\title{
Paper C
}

\section{ULTRASONIC FLOW Metering ERrors DUE TO Pulsating Flow}

\author{
J. Berrebi ${ }^{a}$, P.-E. Martinsson ${ }^{a}$, M. Willatzen ${ }^{b}$, and J. Delsing ${ }^{a}$ \\ ${ }^{a}$ EISLAB, Dept. of Computer Science and Electrical Engineering \\ Lulea University of Technology, SE-971 87 Luleå, Sweden \\ ${ }^{b}$ Mads Clausen Institute for Product Innovation, University of Southern Denmark, \\ Grundtvigs Alle 150, DK-6400 Sønderborg, Denmark
}

Transit-time ultrasonic flow meters present some advantages over other flow meters for district heating industries. They are both accurate and non-intrusive. It is well-known that ultrasonic flow meters are sensitive to installation effects. Installation effects could be static or dynamic. Among the possible dynamic installation effects is pulsating flow. The influence of pulsating flow on the prediction and the zero-crossing operations is investigated. Expressions are found for the prediction error and the zero-crossing error. The relative errors due to the prediction and the zero-crossing are plotted. The prediction error can reach dramatic values while the zero-crossing operation is hardly influenced by flow pulsations.

Keywords: Ultrasonic flow meter; Pulsating flow, Zero-crossing, Installation effect, Prediction error.

\section{C.1 Introduction}

The perturbations due to devices like pumps, compressors and fast acting valves in a flow system will impose so called dynamic installation effects to flow meters. This problem is known from the beginning of the twentieth century but 


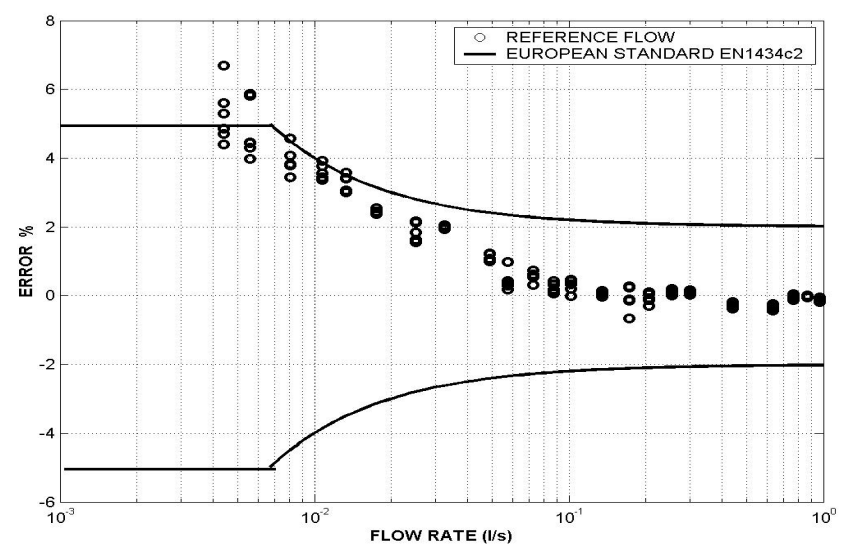

Figure C.1: The flow meter accuracy when there is no pulsating flow. The Error from the meter is compared to the European standard EN 1434-1 [7] showing the maximal permissible error (MPE). The standard's requirements are respected.

is still a major source of error to flow measurements. Pulsating flows generate errors in flow meters such as response-time errors, resonance errors, velocity profile errors, sampling errors, etc ([1], [2], [3]). The errors generated depend on the type of flow meter used e.g. Pitot tubes, vane-type anemometers, inductive flow meters, etc. In district heating and gas applications, the use of ultrasonic flow meters has been dramatically increasing for the last decade. Around $30 \%$ in Sweden and almost $100 \%$ in Denmark of the flow meters currently used in the district heating industry are of ultrasonic type. Ultrasonic flow meters have a high accuracy in stationary flow conditions [4]. Their maximum error does not exceed $2 \%$ or $3 \%$ at turbulent flow rate $(R e>4000)$, and $5 \%$ at laminar $(R e<2000)$ or transient $(2000<R e<4000)$ flow rates (fig.C.1). However, their main drawback is their great sensitivity to installation effects that generate variations in the velocity profile of the flow. A pulsating flow is a dynamic installation effect. In a pulsating flow system, the velocity profile undergoes a dramatic change during one cycle of pulsation compared to a stationary system [2]. This effect generates two errors (fig.C.2) that makes the choice of an appropriate calibration factor complicated ([1], [4]). The inappropriate calibration factor modulates the value of the two precedent errors in an uncontrollable way (fig.C.3). Sampling and zero-crossing methods are analysed here in order to study the effects of pulsations on ultrasonic flow meter performance. 


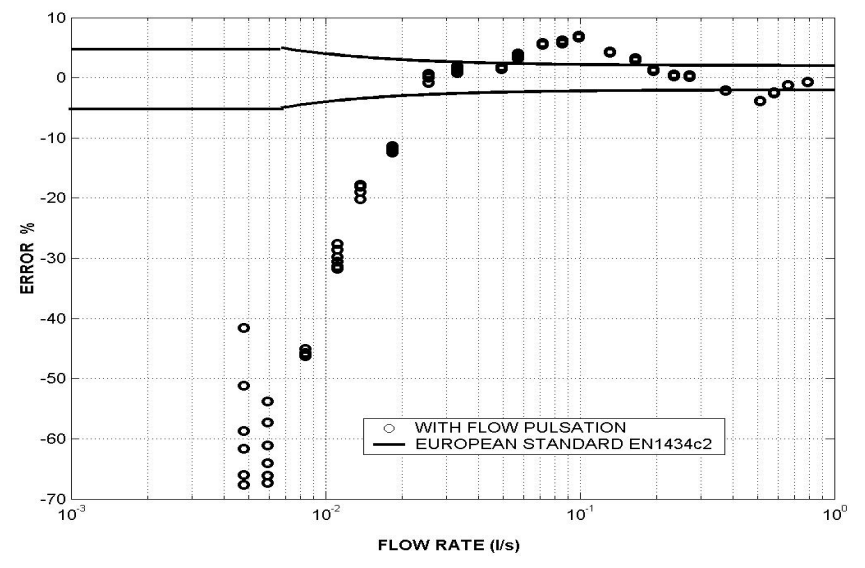

Figure C.2: The flow meter accuracy when pulsations are involved. The Error from the meter is compared to the European standard EN 1434-1 [7] showing the maximal permissible error (MPE). The standard's requirements are not respected for all flows.

\section{C.2 Theory}

\section{C.2.1 Description and principle of the ultrasonic flow me- ter}

An ultrasonic flow meter is described with the help of fig. C.4. For convenience, the flow meter configuration investigated is longitudinal. A similar analysis could be done for diagonal ultrasonic flow meters. The radius of the flow meter body is $R$ and the radius of the ultrasonic transducers is $R_{t d}$. The length between the two transducers is $L$. The estimation of the mean flow velocity is divided in two steps. In the first step, the upstream transducer sends an ultrasonic pulse received by the downstream transducer after time $T_{d w}$. In a first approximation, the pulse velocity is the sum of the speed of sound in water $c$ and the mean flow velocity $\bar{v}$. In the second step, the roles of the transducers are inverted and the upstream transducer receives a pulse travelling with the velocity $c-\bar{v}$ after time $T_{u p}$. A system of two equations is then obtained. Its solution gives an estimation of the mean flow velocity [4]:

$$
\begin{gathered}
\hat{\hat{v}}(t)=\frac{L}{2}\left(\frac{1}{\hat{T}_{d w}\left(t-T_{s}\right)}-\frac{1}{\hat{T}_{u p}(t)}\right)=\frac{L}{2} \frac{\delta T}{\hat{T}_{u p}(t) \hat{T}_{d w}\left(t-T_{s}\right)} \\
\delta T=\hat{T}_{u p}(t)-\hat{T}_{d w}\left(t-T_{s}\right),
\end{gathered}
$$

where $\hat{T}_{d w}\left(t-T_{s}\right)$ and $\hat{T}_{u p}(t)$ are the estimations of the downstream transit-time and the upstream transit-time respectively. The transit-times $T_{d w}$ and $T_{u p}$ can be measured using different techniques, among which are the cross-correlation 


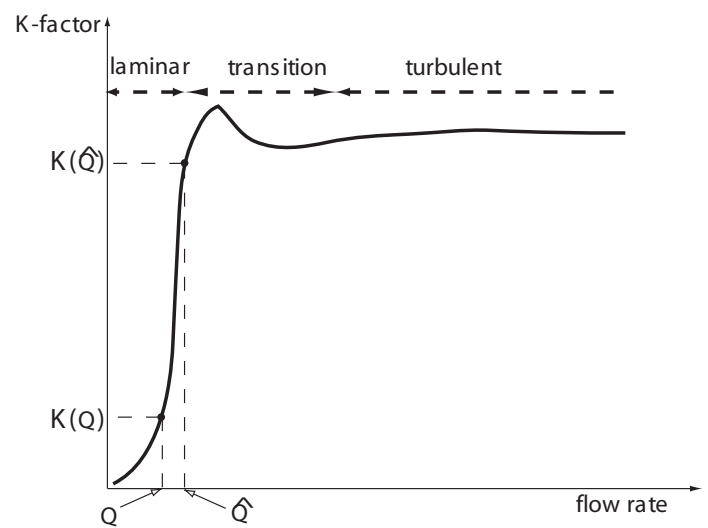

Figure C.3: Amplification of the error by the k-curve. The k-curve indicates the suitable calibration factor $K(\hat{Q})$ for the estimated flow rate $\hat{Q}$. The first estimation of the flow rate is then modulated by the k-factor. The final estimation becomes: $K(\hat{Q}) \hat{Q}$. A small error on the first estimation can induce a large error on the determination of the k-factor. By transitivity, it induces a large error on the final estimation of the flow rate.

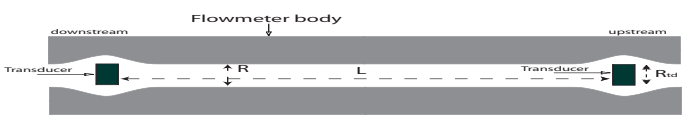

Figure C.4: Axial transit-time ultrasonic flow meter. In the present computations, $R=0.5 \mathrm{~cm}$ and $L=10 \mathrm{~cm}$. 
technique and the zero-crossing technique. The cross-correlation technique is a digital technique that requires the sampling of the pulses and heavy calculations. It becomes consequently too expensive for industrial use. The zero-crossing technique is an analogue technique for determining the transit-times. The zerocrossing technique has a low cost compared to the cross-correlation technique and is therefore widely used in the industry. In the following, the estimator of the transit-times are defined by:

$$
\begin{aligned}
& \hat{T}_{u p}=\frac{T_{N u p}}{N} \\
& \hat{T}_{d w}=\frac{T_{N d w}}{N},
\end{aligned}
$$

where $T_{N u p}$ and $T_{N d w}$ are the times during which $N$ pulses propagate upstream or downstream respectively. Usually, transit-time technique is performed with $N=1$. When $N>1$, the technique is called Sing-Around [5]. $T_{u p}$ and $T_{d w}$ are sampled alternately and periodically. Their sampling period is $2 T_{s}$ where $T_{s}$ is slightly longer than $T_{N u p}$ (and consequently longer than $T_{N d w}$ ). The estimation of $\bar{v}$ made in (C.1) at time $t$ becomes then at time $t+T_{s}$ :

$$
\hat{\bar{v}}\left(t+T_{s}\right)=\frac{L}{2}\left(\frac{1}{\hat{T}_{d w}\left(t+T_{s}\right)}-\frac{1}{\hat{T}_{u p}(t)}\right) .
$$

Each estimation of $T_{u p}$ and $T_{d w}$ is used twice. They are alternately introduced in relations (C.1) and (C.4). Thereby $\bar{v}$ can be estimated at rate $1 / T_{s}$. The fact that the estimations of $T_{u p}$ and $T_{d w}$ are not simultaneous has an influence on the estimation of $\bar{v}$ [4]. This influence is studied in the next paragraph.

\section{C.2.2 The prediction Error}

The sampling of the mean flow velocity is affected by the flow pulsations especially when the time delay $T_{s}$ between the measurement of the upstream transit-time $T_{u p}$ and the measurement of the downstream transit-time $T_{d w}$ is in the same order of magnitude as the period $T_{p}$ of the flow pulsation. The error $E_{s}$ is caused by the prediction error on $T_{u p}$ or $T_{d w}$ and is therefore abusively called prediction error on the flow rate. The difference in transit-times at time $\mathrm{t}$ can be written as:

$$
\delta T(t)=T_{u p}(t)-T_{d w}(t) .
$$

However, as it is technically impossible to estimate both $T_{u p}$ and $T_{d w}$ at time $t$, the estimation of $\delta T$ is performed as follows:

$$
\hat{\delta T}=T_{u p}(t)-T_{d w}\left(t-T_{s}\right) .
$$

The prediction error on $\delta T$ is then:

$$
E_{\delta T}=T_{d w}(t)-T_{d w}\left(t-T_{s}\right)
$$


Denoting by $T_{d}=\frac{L}{c}$ the zero-flow transit-time between the two transducers and by $\bar{v}_{p}(t)$ the mean flow velocity at time $t$ when pulsations are involved, the expression of $E_{\delta T}$ can be developed as follows by using (C.1) and (C.4) in (C.7):

$$
E_{\delta T}=\frac{L}{c\left(1+\frac{\bar{v}_{p}(t)}{c}\right)}-\frac{L}{c\left(1+\frac{\bar{v}_{p}\left(t-T_{s}\right)}{c}\right)} .
$$

Since $\bar{v}_{p} \ll c$, the Taylor expansion of (C.8) gives:

$$
\begin{aligned}
E_{\delta T} & \simeq T_{d}-\frac{L}{c^{2}} \bar{v}_{p}(t)-\left(T_{d}-\frac{L}{c^{2}} \bar{p}_{p}\left(t-T_{s}\right)\right) \\
& \simeq \frac{L}{c^{2}}\left(\bar{v}_{p}\left(t-T_{s}\right)-\bar{v}_{p}(t)\right)
\end{aligned}
$$

The prediction error $E_{\delta T}$ made on the estimation of $\delta T$ leads then to the relative prediction error $E_{s}$ on the mean flow velocity estimation:

$$
E_{s}=\frac{\bar{v}_{p}\left(t-T_{s}\right)-\bar{v}_{p}(t)}{\bar{v}_{p}(t)}
$$

\section{C.2.3 Zero-crossing error}

The upstream and downstream transit times are very often measured by zerocrossing techniques. In order to give a description of the zero-crossing technique, the transformation between electric and pressure signals is assumed to be linear with gain one. This assumption will not lead to qualitatively different results in terms of flow measurement properties. A pulse $p_{u}^{(1)}$ is sent by the emitting transducer and received by the receiving transducer at time $t_{0}=0$. This pulse is composed of 5 periods of a sinusoid of frequency $f_{u}=1 / T_{u}$ damped by an exponential window. The damping in water is neglected. Diffraction is not taken into account for convenience. As the propagation model studied in the precedent paragraph is linear and non-dissipative, the received signal can be written as a translation in time of the pulse sent.

$$
\begin{aligned}
& \forall t \in\left[t_{0}, t_{0}+5 T_{u}\right], \quad p_{u}^{(1)}(t)=p_{u}^{0} \sin \left(2 \pi f_{u}\left(t-t_{0}\right)\right) \exp \left(-\frac{t-t_{0}}{T_{u}}\right) \\
& \forall t \notin\left[t_{0}, t_{0}+5 T_{u}\right], \quad p_{u}^{(1)}(t)=0
\end{aligned}
$$

Assuming that the first pulse is sent independently of the flow pulsations, the flow pulsations have a phase $2 \pi f_{u} \theta$ that is a free parameter since $t_{0}=0$ is defined by $p_{u}^{(1)}$ :

$$
\forall t \in R, p_{p}(t)=p_{p}^{0} \sin \left(2 \pi f_{u}(t-\theta)\right) .
$$

The value of the total pressure at time $t_{0} p_{p}\left(t_{0}\right)+p_{u}^{(1)}\left(t_{0}\right)$ is memorized as the threshold for the zero-crossing. This is in fact simply $p_{p}\left(t_{0}\right)$ since $p_{u}^{(1)}\left(t_{0}\right)=0$. Simultaneously as $p_{u}^{(1)}$ is being received, a second pulse is sent from the same 


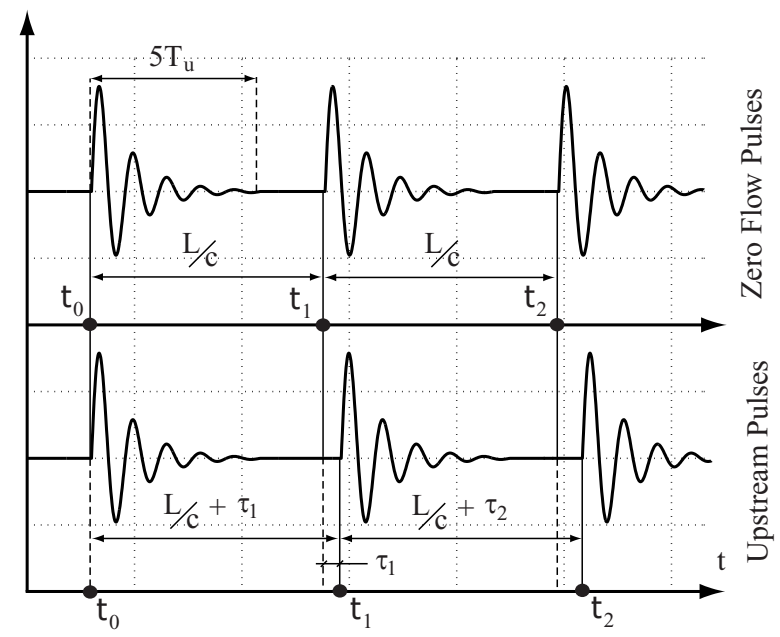

Figure C.5: Comparison between zero-crossing at zero flow and at non-zero flow. On the upper part of the figure, a pulse is received every $t_{j}=j L / c$ since the mean flow velocity is zero. In the lower part of the figure, flow pulsations are involved. They induce positive (resp. negative) extensions $\tau_{j}$ of the upstream (resp. downstream) transit-time.

emitter. This process is repeated $N-1$ times.

$$
\forall j \in[1, N-1],\left\{\begin{array}{l}
\tau_{0}=0 \\
(j \neq 0) \Rightarrow \tau_{j}=\frac{L}{c^{2}} \bar{v}_{p}\left(t_{j-1}\right) \\
t_{j}=j T_{d}+\sum_{k=0} \tau_{k} \\
\forall t \in\left[t_{j}, t_{j}+5 T_{u}\right], \\
p_{u}^{(j+1)}(t)=p_{u}^{0} \sin \left(2 \pi f_{u}\left(t-t_{j}\right)\right) \exp \left(-\frac{t-t_{j}}{T_{u}}\right) \\
\forall t \notin\left[t_{j}, t_{j}+5 T_{u}\right], \quad p_{u}^{(j+1)}(t)=0
\end{array}\right.
$$

where $\tau_{j}$ denote the difference between the current transit-time (when the flow velocity is $\left.\bar{v}_{p}\left(t_{j-1}\right)\right)$ and the zero-flow transit-time $T_{d}$ (fig.C.5). From time $t_{j-1}$, a trigger is looking for the first time when the received pressure $p_{p}(t)+p_{u}^{(j)}(t)$ reaches the threshold $p_{p}\left(t_{0}\right)$. The trigger is looking for the first zero of the signal $p_{p}(t)+p_{u}^{(j)}(t)-p_{p}\left(t_{0}\right)$.

$$
\begin{aligned}
& p_{p}(t)+p_{u}^{(j+1)}(t)-p_{p}\left(t_{0}\right)=0 \\
& t \in\left[t_{j}, t_{j}+T_{u}\right] .
\end{aligned}
$$

\section{C.2.3.1 Constant mean flow velocity}

If the flow velocity is constant, then $p_{p}$ is also constant, equal to the threshold $p_{p}\left(t_{0}\right)$, and $\tau_{j}=\tau$. The trigger simply determines the first zero $z_{j}$ of each 
pressure pulse $p_{u}^{(j)}$. Then $z_{j+1}$ is the solution of:

$$
\begin{aligned}
& p_{u}^{(j+1)}(t)=0 \\
& t \in\left[t_{j}, t_{j}+T_{u}\right] .
\end{aligned}
$$

Solving for (C.17) using (C.15) leads to:

$$
\begin{gathered}
(C .17) \Longleftrightarrow\left\{\begin{array}{c}
\sin \left(2 \pi f_{u}\left(t-t_{j}\right)\right)=0 \\
0<t-t_{j}<T_{u}
\end{array}\right. \\
z_{j+1}=t_{j}+\frac{T_{u}}{2} .
\end{gathered}
$$

The transit time $T_{j}$ is then determined by the following formula:

$$
\begin{gathered}
\forall j \in[2, N], T_{j}=z_{j}-z_{j-1}=t_{j-1}-t_{j-2} \\
\forall j \in[2, N], T_{j}=T_{d}+\tau .
\end{gathered}
$$

A reduction of the noise present in the measurement is performed by taking the average of all $T_{j}$. The estimation of the transit time is then:

$$
\begin{gathered}
\hat{T}=\frac{1}{N-1} \sum_{j=2}^{N} T_{j} \\
\hat{T}=T_{d}+\tau .
\end{gathered}
$$

\section{C.2.3.2 Sinusoidal mean flow velocity}

If $\bar{v}_{p}$ is slowly varying over the duration of $\mathrm{N}$ pulses, the zero-crossing found in (C.19) is slightly delayed by $\varepsilon_{j}$ (fig.C.6):

$$
p_{u}^{(j)}\left(z_{j}+\varepsilon_{j}\right)+p_{p}\left(z_{j}+\varepsilon_{j}\right)-p_{p}\left(t_{0}\right)=0 .
$$

Assuming that $\left|\varepsilon_{j}\right| \ll z_{j}$ (assumption $H_{1}$ ) and applying Taylor's formula to (C.24) yields:

$$
p_{u}^{(j)}\left(z_{j}\right)+\frac{d p_{u}^{(j)}}{d t}\left(z_{j}\right) \varepsilon_{j}+p_{p}\left(z_{j}\right)+\frac{d p_{p}}{d t}\left(z_{j}\right) \varepsilon_{j}-p_{p}\left(t_{0}\right) \simeq 0 .
$$

From (C.17), we have $p_{u}^{(j)}\left(z_{j}\right)=0$. Moreover, if $\left|\frac{d p_{p}}{d t}\left(z_{j}\right)\right| \ll\left|\frac{d p_{u}^{(j)}}{d t}\left(z_{j}\right)\right|$ (assumption $H_{2}$ ), $\varepsilon_{j}$ becomes:

$$
\varepsilon_{j}=\frac{p_{p}\left(t_{0}\right)-p_{p}\left(z_{j}\right)}{\frac{d p_{u}^{(j)}}{d t}\left(z_{j}\right)}
$$

Inserting (C.14) and (C.15) into equation (C.26) yields:

$$
\varepsilon_{j} \simeq \frac{p_{p}^{0}}{p_{u}^{0}} \frac{\sin \left(2 \pi f_{p}\left(t_{0}-\theta\right)\right)-\sin \left(2 \pi f_{p}\left(z_{j}-\theta\right)\right)}{f_{u}\left(2 \pi \cos \left(2 \pi f_{u} \frac{T_{u}}{2}\right)-\sin \left(2 \pi f_{u} \frac{T_{u}}{2}\right)\right) \exp (-1 / 2)},
$$


that is in fact:

$$
\varepsilon_{j} \simeq \frac{p_{p}^{0}}{p_{u}^{0}} \frac{\sin \left(2 \pi f_{p}(-\theta)\right)-\sin \left(2 \pi f_{p}\left(z_{j}-\theta\right)\right)}{f_{u}(-2 \pi \exp (-1 / 2))} .
$$

The transit-time and the average transit-time then become:

$$
\begin{gathered}
\forall j \in[2, N], T_{j}=z_{j}+\varepsilon_{j}-z_{j-1}-\varepsilon_{j-1}=T_{d}+\tau_{j-1}+\left(\varepsilon_{j}-\varepsilon_{j-1}\right) \\
\hat{T}=T_{d}+\frac{1}{N-1} \sum_{i=1}^{N-1} \tau_{i}+\bar{\varepsilon} \\
\bar{\varepsilon}=\frac{1}{N-1}\left(\varepsilon_{N}-\varepsilon_{1}\right)
\end{gathered}
$$

With help of (C.28), $\bar{\varepsilon}$ becomes:

$$
\begin{gathered}
\bar{\varepsilon} \simeq \frac{p_{p}^{0}}{p_{u}^{0}} \frac{\sin \left(2 \pi f_{p}\left(z_{1}-\theta\right)\right)-\sin \left(2 \pi f_{p}\left(z_{N}-\theta\right)\right)}{(N-1) f_{u}(-2 \pi \exp (-1 / 2))} \\
\bar{\varepsilon} \simeq \frac{p_{p}^{0}}{p_{u}^{0}} \frac{-2 \cos \left(2 \pi f_{p}\left(\frac{z_{1}+z_{N}}{2}-\theta\right)\right) \sin \left(2 \pi f_{p}\left(z_{N}-z_{1}\right)\right)}{(N-1) f_{u}(-2 \pi \exp (-1 / 2))}
\end{gathered}
$$

As $\theta$ is a free parameter, it can be changed to $\frac{z_{1}+z_{N}}{2}-\theta$. Moreover, assuming that $f_{p}\left(z_{N}-z_{1}\right) \simeq f_{p}(N-1) T_{d} \ll 1$ leads to:

$$
\bar{\varepsilon} \simeq \frac{\cos \left(2 \pi f_{p} \theta\right)}{\exp (-1 / 2)} \frac{p_{p}^{0}}{p_{u}^{0}} \frac{f_{p}}{f_{u}} T_{d}=\bar{\varepsilon}^{0} \cos \left(2 \pi f_{p} \theta\right) .
$$

Notice that the latter is valid if and only if there is solution for the zero-crossing. If the threshold does not cross the pulse ( $p_{u}^{(0)}$ too small), formula (C.34) has no meaning. The problem has a solution if

$p_{u}^{(0)}>\left|\frac{d p_{p}}{d t}\right|_{\text {Max }} N T_{d}=2 N T_{d} \pi f_{p} p_{p}^{0}$

\section{C.2.3.3 Filtering the flow pulsations}

By applying a high-pass filter on the total received pressure (before zero-crossing), it is easier to have a solution of form (C.34) to the problem posed in (C.24). By using a first order filter:

$$
H(\omega)=H_{0} \frac{i \omega / \omega_{c}}{1+i \omega / \omega_{c}}
$$

where $H_{0}=1$, the pressure from the flow pulsations is damped by a factor $H\left(\omega_{p}\right)\left(\omega_{p}=2 \pi f_{p}\right)$. Then replacing $p_{p}^{0}$ in (C.34) by $H\left(\omega_{p}\right) p_{p}^{0}$ gives for $p_{u}^{0}>$ $2 N T_{d} \pi f_{p} H\left(\omega_{p}\right) p_{p}^{0}$ (assumption $H_{3}$ ):

$$
\bar{\varepsilon}_{H} \simeq \frac{\cos \left(2 \pi f_{p} \theta\right)}{\exp (-1 / 2)} \frac{H\left(\omega_{p}\right) p_{p}^{0}}{p_{u}^{0}} \frac{f_{p}}{f_{u}} T_{d}=\bar{\varepsilon}_{H}^{0} \cos \left(2 \pi f_{p} \theta\right) .
$$




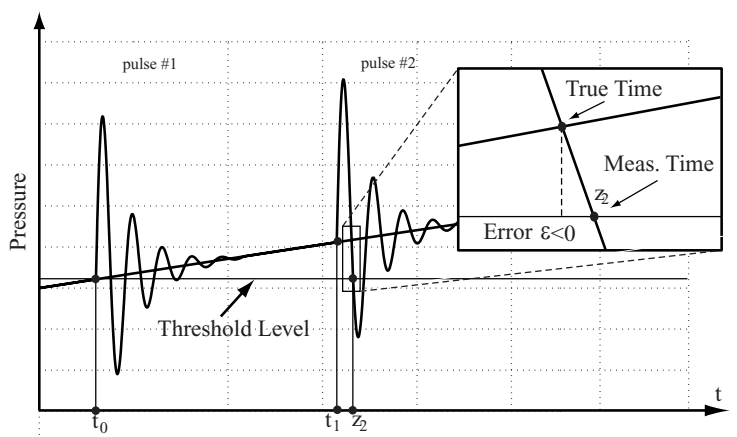

Figure C.6: Error induced by a pulsating flow on the zero-crossing process. The threshold level is given by the value of the pressure at time $t=t_{0}$. From that moment, the detector is looking for the first time when the pulses cross the threshold. The measured zero are then given by the $z_{j}$. As the flow pulsations are due to variation of the pressure $p_{p}(t)$, they induce an error denoted by $\epsilon$. When the pressure is increasing (case of the figure), the threshold becomes lower than it should be relatively to the pulse. Then $\epsilon$ is negative. If the pressure is decreasing, $\epsilon$ is positive.

\section{C.2.3.4 Error on the flow measurement}

Assuming now that a downstream transit time is measured at time $\theta$ and that the following upstream transit time is measured at time $\theta+T_{s}$, the estimation of the mean flow velocity according to (C.1) then reads:

$$
\begin{gathered}
\hat{\bar{v}}=\frac{L}{2}\left(\frac{1}{\hat{T}_{d w}(\theta-T s)}-\frac{1}{\hat{T}_{u p}(\theta)}\right)=\frac{L}{2} \frac{\delta T}{\hat{T}_{u p}(\theta) \hat{T}_{d w}(\theta-T s)} \\
\delta T=\hat{T}_{u p}(\theta)-\hat{T}_{d w}(\theta-T s) .
\end{gathered}
$$

Taking the logarithmic differentiate of (C.1) and focusing only on the zerocrossing terms leads to:

$$
E_{z}=\frac{\triangle \bar{v}}{\bar{v}}=\frac{\bar{\varepsilon}_{H}(\theta)-\bar{\varepsilon}_{H}(\theta-T s)}{\delta T}-\frac{\bar{\varepsilon}_{H}(\theta)}{T_{u p}(\theta)}-\frac{\bar{\varepsilon}_{H}(\theta-T s)}{T_{d w}\left(\theta-T_{s}\right)} .
$$

By neglecting the two latter terms, the relative error on the flow velocity becomes:

$$
E_{z} \simeq \frac{\bar{\varepsilon}_{H}(\theta)-\bar{\varepsilon}_{H}(\theta-T s)}{\delta T} .
$$

As $\delta T \simeq \frac{L \bar{v}_{p}(\theta)}{c^{2}}, E_{z}$ becomes:

$$
E_{z} \simeq \frac{c^{2}}{L \bar{v}_{p}(\theta)}\left(\bar{\varepsilon}_{H}(\theta)-\bar{\varepsilon}_{H}(\theta-T s)\right) .
$$


Developing the latter expression leads to:

$$
E_{z} \simeq \frac{c^{2} \bar{\varepsilon}_{H}^{0}}{L \bar{v}_{p}^{0}} \frac{\left(\cos \left(2 \pi f_{p} \theta\right)-\cos \left(2 \pi f_{p}(\theta-T s)\right)\right)}{\sin \left(2 \pi f_{p} \theta\right)} .
$$

\section{C.3 Results/Discussion}

The expressions of $E_{s}$ and $E_{z}$ respectively found in (C.11) and in (C.42) are suitable for both liquids and gases. Both $E_{s}$ and $E_{z}$ cannot be overestimated since the sinusoids present in the quotient of (C.11) and of (C.42) can be in principle equal to zero. In practice, the probability that $\theta$ will be equal to (or in the neighbourhood of) $0 \bmod \frac{T_{p}}{2}$ is small. The sinusoid present in the quotient of (C.11) and of (C.42) will not be equal to zero.

\section{C.3.1 Prediction error}

A typical value for the flow pulsations amplitude is $\bar{v}_{p}^{0}=1 \mathrm{~m} / \mathrm{s}$. By choosing different values for the number $N$ of loops and for the pulsations frequency $f_{p}, E_{s}$ can vary considerably. In fig.C.7 and fig.C.8 are respectively plotted the prediction error for $N=2$ and for $N=100$ for $f_{p}=10 \mathrm{~Hz}$. These figures shows that $E_{s}$ can easily reach around $10 \%$ when there is no averaging of the transittimes $(N=2)$. The same error can reach dramatic values when averaging the transit-times $(N=100)$. This is due to the fact that when $N$ increases, the sampling interval also increases leaving a longer time interval to the variations of the flow velocity. There is a compromise to find between $N$ and $f_{p}$. When $f_{p}$ reaches high values, it is wiser to take $N$ smaller.

\section{C.3.2 Zero-crossing in water applications}

The flow pulsations in water appears mostly in a range of frequencies between $0.1 \mathrm{~Hz}$ and $100 \mathrm{~Hz}$. The pressure is of the order of $1 \mathrm{bar}$. the central frequency $f_{u}$ of the pulse is between $0.5 \mathrm{MHz}$ and $4 \mathrm{MHz}$. The ultrasonic pressure varies in a range between $1 P a$ and $1000 P a$. A typical example is given for flow pulsations of amplitude $1 \mathrm{~Pa}$ and of central frequency $10 \mathrm{~Hz}$. Taking $p_{u}^{0}=$ $1000 \mathrm{~Pa}, f_{u}=4 \mathrm{MHz}, f_{p}=10 \mathrm{~Hz}$ and $N=2$, leads to the graph plotted on fig.C.9. Calculating the transit-times with $N=100$ gives the curve plotted on fig.C.10. The frequency $f_{u}$ of the ultrasonic pulse is so much higher than $f_{p}$ that the filter erase almost totally the flow pulsations. That is why the zero-crossing errors found have no significance.

\section{C.3.3 Zero-crossing in gas applications}

Flow pulsations in gas can in practice reach higher frequencies, up to $1 \mathrm{kHz}$. The pulse central frequency is usually lower than in liquids. $f_{u}$ is then confined between $100 \mathrm{kHz}$ and $1 \mathrm{MHz}$. One can imagine that by choosing a high flow 


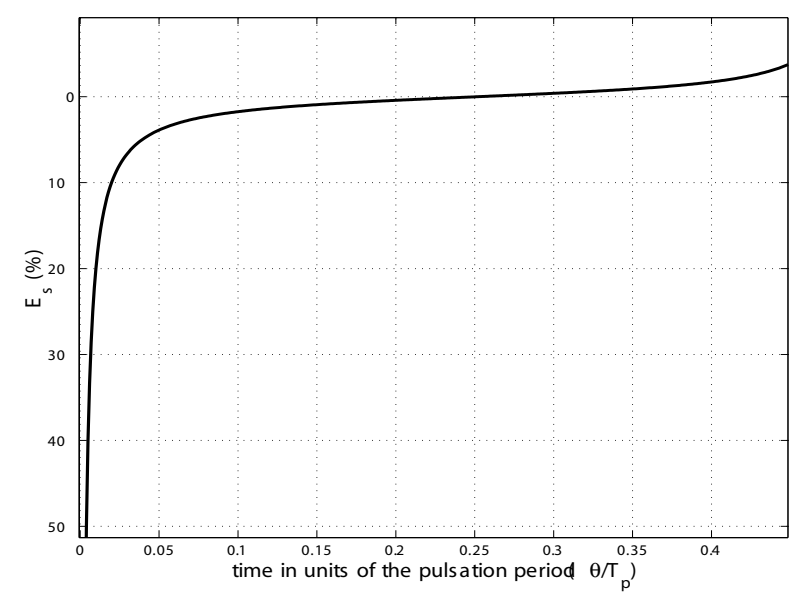

Figure C.7: The variations of the prediction error $E_{s}$ over time when $N=2$. The maximal error can easily reach $50 \%$ (C.2.2).

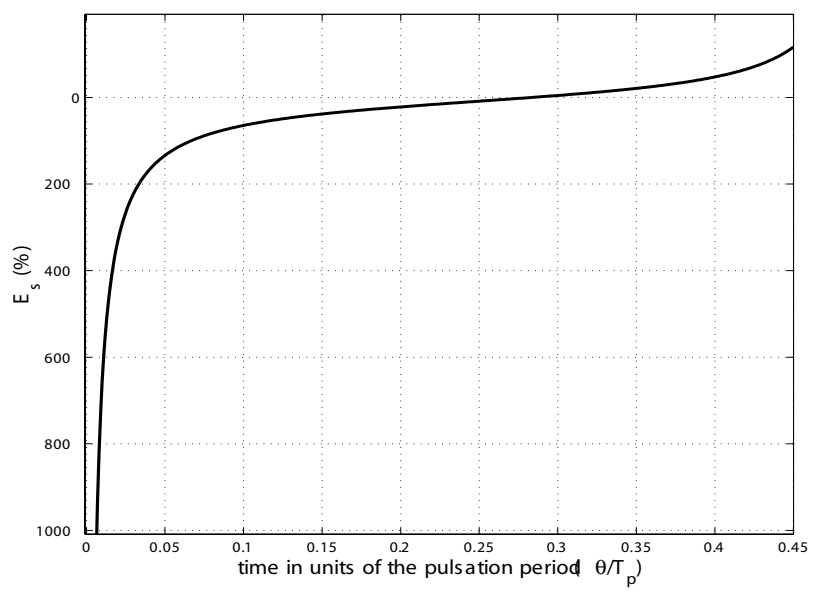

Figure C.8: The variations of the prediction error $E_{s}$ over time when $N=100$. The maximal error can easily exceed $100 \%$. The error is increased by the high number of pulses sent. The sampling time is too long, inducing a large prediction error (C.2.2) 


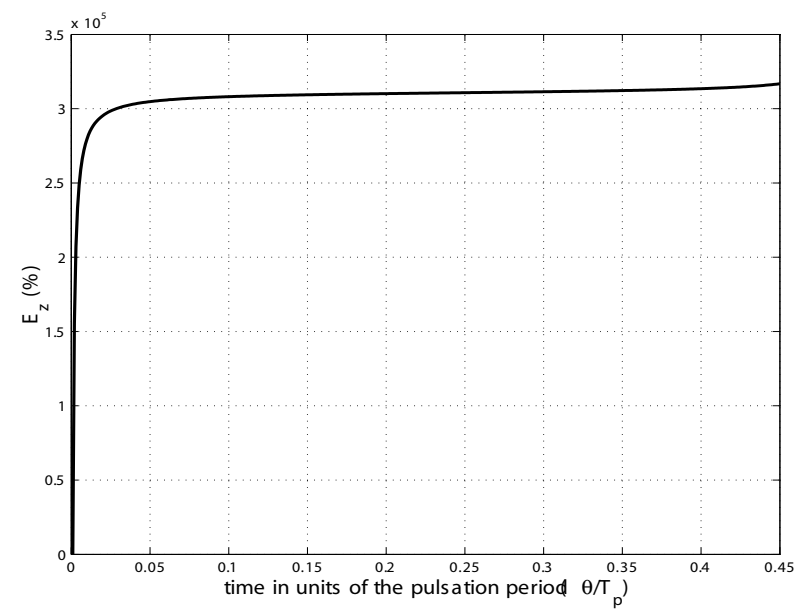

Figure C.9: The variations of the zero-crossing error $E_{z}$ over time when $N=2$. The maximal error does not exceed $4.10^{-5} \%$

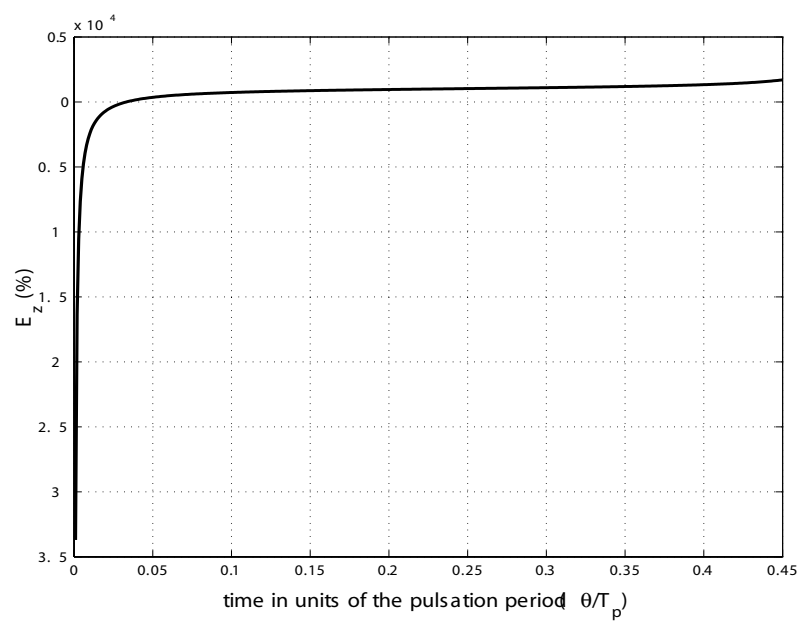

Figure C.10: The variations of the zero-crossing error $E_{z}$ over time when $N=$ 100. The maximal error does not exceed $2 \cdot 10^{-4 \%}$. It shows that the flow pulsations do not have any influence on the zero-crossing process. 
pulsation frequency of $1 \mathrm{kHz}$ and a low pulse central frequency of $100 \mathrm{kHz}$, flow pulsations would still be present after filtering. Since the speed of sound in air is only $330 \mathrm{~m} / \mathrm{s}$, the time of travel $T_{d}$ is equal to $0.3 \mathrm{~ms}$, the flow can no more be considered as static during the time of propagation and it is not valid to apply the model employed in the present paper. In our study, flow pulsations cannot reach higher frequencies than $100 \mathrm{~Hz}$. Moreover, since the damping is much stronger in gas than in liquids, the amplitude of the ultrasonic pressure $p_{u}^{0}$ at the receiver will be sensibly lower than at the emitter. All these considerations make assumptions $\mathrm{H}_{2}$ and $\mathrm{H}_{3}$ more difficult to satisfy for gases. However, these assumptions are satisfied in practice by filtering more efficiently flow pulsations. It implies to use a high-pass filter of higher order than $H$. This is usually the case in the industrial applications. It is then possible to reach the same level of error than in liquids.

\section{C.4 Conclusion}

The zero-crossing method used for measuring the upstream and downstream transit-times is not sensitive to flow pulsations. Indeed, an appropriate filter can remove the flow pulsations before operating the zero-crossing. However, the sampling of the flow velocity cannot be perfect in the way that upstream and downstream transit-times cannot be measured simultaneously. A prediction error depending on the time position, the pulsations frequency and the number of loops used for averaging the transit-times can then reach dramatic values. Moreover, the present work does not deal with the possible influence of the flow pulsations on the ultrasonic propagation due to velocity profile variations [6]. So far, no propagation model relevant for the effects caused by the pulsations has been found. But one has to be aware that such errors probably exist. 


\section{Bibliography}

[1] A. Hayward, Flowmeters, London: The MacMillan Press Ltd (1979), pp. $112-116$.

[2] E. Håkansson, J. Delsing, Effects of Pulsating Flow on an Ultrasonic Gas Flowmeter, Lund,Sweden: Lund Institute of Technology (1993).

[3] C. Carlander, Installation Effect and Self-Diagnostics for Ultrasonic Flow Measurement. Luleå, Sweden: Luleå University of Technology (2001).

[4] L. Lynnworth, A. Brown, Ultrasonic Flow meters. Flow Measurement, Second edition, Research Triangle Park: D.W. Spitzer, ISA (2001).

[5] J. Delsing, US patent 5796 009, Method for measuring in a fluid with the aid of sing-around technique.

[6] Uchida S. , The Pulsating Viscous Flow Superposed on the Steady Laminar Motion of an incompressible Fluid in a Circular Pipe, Zamp (1956), pp. 403-421.

[7] European Committee for Standardisation (CEN) and Swedish Standard Institution (SIS). Heat meters-part 1: General requirements. EN 1434-1 (1997). 